\title{
Dynamics of thermal Casimir-Polder forces on polar molecules
}

\author{
Simen Ådnøy Ellingsen \\ Department of Energy and Process Engineering, Norwegian University of Science and Technology, N-7491 Trondheim, Norway \\ Stefan Yoshi Buhmann and Stefan Scheel \\ Quantum Optics and Laser Science, Blackett Laboratory, Imperial College London, \\ Prince Consort Road, London SW7 2AZ, United Kingdom
}

(Dated: October 25, 2018)

\begin{abstract}
We study the influence of thermal Casimir-Polder forces on the near-surface trapping of cold polar molecules, with emphasis on $\mathrm{LiH}$ and $\mathrm{YbF}$ near an Au surface at room temperature. We show that even for a molecule initially prepared in its electronic and rovibrational ground state, the Casimir-Polder force oscillates with the molecule-wall separation. The non-resonant force and the evanescent part of the resonant force almost exactly cancel at high temperature which results in a saturation of the (attractive) force in this limit. This implies that the Casimir-Polder force on a fully thermalised molecule can differ dramatically from that obtained using a naïve perturbative expansion of the Lifshitz formula based on the molecular ground-state polarisability. A dynamical calculation reveals how the spatial oscillations die out on a typical time scale of several seconds as thermalisation of the molecule with its environment sets in.

PACS numbers: $34.35 .+\mathrm{a}, 12.20 .-\mathrm{m}, 42.50 . \mathrm{Ct}, 42.50 . \mathrm{Nn}$
\end{abstract}

\section{INTRODUCTION}

Cold ensembles of polar molecules such as YbF have recently received particular attention due to their potential use as ultra-sensitive probes of the permanent electric dipole moment of the electron [1], measurements of which allow for investigating the possible existence of physics beyond the standard model [2]. The need for longer interrogation times has led to the development of Stark deceleration techniques for these heavy molecules [3, 4], with a view to ultimately be able to trap molecules near microstructured surfaces (chips). Recently, trapping of light molecules such as metastable $\mathrm{CO}$ in travelling potential wells near a chip surface was achieved [5]. Another light diatomic molecule that has received considerable attention due to its large dipole moment is $\mathrm{LiH}$; and the production of supersonic beams of cold $\mathrm{LiH}$ has been reported [6].

When attempting to trap polar molecules in close proximity to a surface, attractive Casimir-Polder (CP) forces [7] - effective electromagnetic forces between a neutral and polarisable particle and a macroscopic object - need to be taken into account as an important limiting factor. Thermal CP forces on atoms at thermal equilibrium with both the electromagnetic field and the present macroscopic bodies have been intensively studied in the past on the basis of Lifshitz theory [8 11], linear response theory [12, 13] or normal-mode techniques [14, 15]. At room temperature, the energies associated with atomic transitions are much larger than the thermal energy, $\hbar \omega_{A} \gg k_{\mathrm{B}} T$, resulting in very low thermal photon numbers. A "high-temperature limit" is only accessible in a geometric sense when the atom-surface separation $z_{A}$ is much larger than the thermal wavelength, $z_{A} \gg \hbar c /\left(2 \pi k_{\mathrm{B}} T\right)$; in this case the thermal CP force on the atom can be approximated by [12, 13]

$$
\boldsymbol{F}\left(\boldsymbol{r}_{A}\right) \approx-\frac{\left|\boldsymbol{d}_{A}\right|^{2}}{8 \pi \varepsilon_{0} z_{A}^{4}} \frac{k_{\mathrm{B}} T}{\hbar \omega_{A}} \boldsymbol{e}_{z}
$$

for a two-level atom (transition frequency $\omega_{A}$, dipole matrix element $\boldsymbol{d}_{A}$ ) interacting with a perfectly conducting plate (unit normal $\boldsymbol{e}_{z}$ ).

The situation is different for molecules: whereas transition energies of atoms are typically much larger than attainable thermal energies, the energies associated with rotational and vibrational transitions of molecules, heavy molecules in particular, are often small compared to the thermal energy even at room temperature. A genuine high-temperature limit $\hbar \omega_{A} \ll k_{\mathrm{B}} T$ is hence realised with an associated large number of thermal photons being present. An additional consequence of the long transition wavelengths is the fact that $\mathrm{CP}$ forces on molecules are expected to have a long range with the non-retarded regime $z_{A} \ll c / \omega_{A}$ extending quite far out from the surface. A naïve application of the above formula (1) for atoms beyond its scope to the high-temperature limit $\hbar \omega_{A} \ll k_{\mathrm{B}} T$ would suggest that the force can get arbitrarily strong for molecules of smaller and smaller transition energies, which already indicates that $\mathrm{CP}$ forces on molecules must be treated with care.

Supersonic beam expansions typically produce cold molecules that are to a large fraction in their rovibrational ground states. For example, in the experiment reported in Ref. [6], $90 \%$ of the observed cold $\mathrm{LiH}$ molecules were in their electronic and rovibrational ground state $X^{1} \Sigma^{+}$. The cold molecule and the room-temperature surface are thus strongly out of equilibrium with respect to each other, so a study of the CP interaction necessitates that account be taken of the full non-equilibrium dynamics of the rotational and vibrational degrees of freedom of the cold molecule coupled to its thermal envi- 
ronment. In contrast, in the context of non-equilibrium forces on thermalised atoms in an environment of nonuniform temperature, as recently proposed [16] and measured [17], a study of the full internal atomic dynamics was not necessary.

In this article, we study the non-equilibrium thermal $\mathrm{CP}$ force on a polar molecule which is initially in its electronic and rovibrational ground state in the vicinity of a metal surface. A recently developed dynamical theory of forces on single atoms or molecules in arbitrary internal states and arbitrary uniform temperature environments [18] provides the necessary framework (note that a similar theory has been developed for two-atom van der Waals forces [19]). In particular, we will show that in contrast to the above intuitive expectation obtained from comparison with the atom case, the attractive $\mathrm{CP}$ force on a molecule saturates in the high-temperature limit.

\section{CASIMIR-POLDER FORCE FOR GIVEN MOLECULAR STATES}

We consider a polar molecule (energy eigenstates $|n\rangle$, eigenenergies $\hbar \omega_{n}$, transition frequencies $\omega_{m n}=\omega_{m}-\omega_{n}$, dipole matrix elements $\boldsymbol{d}_{m n}$ ) which is prepared in an incoherent superposition of its energy eigenstates with probabilities $p_{n}$. As shown in Ref. [18], the thermal CP force on such a molecule is given by

$$
\boldsymbol{F}\left(\boldsymbol{r}_{A}\right)=\sum_{n} p_{n} \boldsymbol{F}_{n}\left(\boldsymbol{r}_{A}\right)
$$

with perturbative force components

$$
\begin{aligned}
\boldsymbol{F}_{n}\left(\boldsymbol{r}_{A}\right) & =-\mu_{0} k_{\mathrm{B}} T \sum_{N=0}^{\infty}\left(1-\frac{1}{2} \delta_{N 0}\right) \xi_{N}^{2} \\
& \times \nabla_{A} \operatorname{Tr}\left[\boldsymbol{\alpha}_{n}\left(\mathrm{i} \xi_{N}\right) \cdot \mathbf{G}^{(1)}\left(\boldsymbol{r}_{A}, \boldsymbol{r}_{A}, \mathrm{i} \xi_{N}\right)\right] \\
+ & \mu_{0} \sum_{k} \omega_{n k}^{2}\left\{\Theta\left(\omega_{n k}\right)\left[n\left(\omega_{n k}\right)+1\right]-\Theta\left(\omega_{k n}\right) n\left(\omega_{k n}\right)\right\} \\
& \times \nabla_{A} \boldsymbol{d}_{n k} \cdot \operatorname{Re} \mathbf{G}^{(1)}\left(\boldsymbol{r}_{A}, \boldsymbol{r}_{A},\left|\omega_{n k}\right|\right) \cdot \boldsymbol{d}_{k n}
\end{aligned}
$$

and molecular polarisability

$$
\boldsymbol{\alpha}_{n}(\omega)=\lim _{\epsilon \rightarrow 0} \frac{1}{\hbar} \sum_{k}\left[\frac{\boldsymbol{d}_{k n} \boldsymbol{d}_{n k}}{\omega+\omega_{k n}+\mathrm{i} \epsilon}-\frac{\boldsymbol{d}_{n k} \boldsymbol{d}_{k n}}{\omega-\omega_{k n}+\mathrm{i} \epsilon}\right] .
$$

Here, $\mathbf{G}^{(1)}$ is the scattering part of the classical Green tensor for the electromagnetic field in the given environment and $\xi_{N}=2 \pi k_{\mathrm{B}} T N / \hbar$ denotes the Matsubara frequencies. The CP force (3) contains both non-resonant contributions (first term) and resonant ones (second term), where the former would also follow from applying Lifshitz theory in conjunction with the ground-state polarisability (we refer to it as Lifshitz-like force in the following) and the latter are due to the absorption and emission of thermal photons with photon number

$$
n(\omega)=\frac{1}{e^{\hbar \omega /\left(k_{\mathrm{B}} T\right)}-1} .
$$

Given a probability distribution $p_{n}$, Eq. (3) allows us to compute the thermal $\mathrm{CP}$ force. In particular, if the molecule is in an isotropic state such as the ground state or a thermal state (see below), the forces simplifies to [18]

$$
\begin{aligned}
& \boldsymbol{F}_{n}\left(\boldsymbol{r}_{A}\right)=-\mu_{0} k_{\mathrm{B}} T \sum_{N=0}^{\infty}\left(1-\frac{1}{2} \delta_{N 0}\right) \xi_{N}^{2} \alpha_{n}\left(\mathrm{i} \xi_{N}\right) \\
& \times \nabla_{A} \operatorname{Tr}\left[\mathbf{G}^{(1)}\left(\boldsymbol{r}_{A}, \boldsymbol{r}_{A}, \mathrm{i} \xi_{N}\right)\right] \\
& +\frac{\mu_{0}}{3} \sum_{k} \omega_{n k}^{2}\left\{\Theta\left(\omega_{n k}\right)\left[n\left(\omega_{n k}\right)+1\right]-\Theta\left(\omega_{k n}\right) n\left(\omega_{k n}\right)\right\} \\
& \quad \times\left|\boldsymbol{d}_{n k}\right|^{2} \nabla_{A} \operatorname{Tr} \operatorname{Re} \mathbf{G}^{(1)}\left(\boldsymbol{r}_{A}, \boldsymbol{r}_{A},\left|\omega_{n k}\right|\right)
\end{aligned}
$$

with

$$
\alpha_{n}(\omega)=\lim _{\epsilon \rightarrow 0} \frac{1}{3 \hbar} \sum_{k}\left[\frac{\left|\boldsymbol{d}_{n k}\right|^{2}}{\omega+\omega_{k n}+\mathrm{i} \epsilon}-\frac{\left|\boldsymbol{d}_{n k}\right|^{2}}{\omega-\omega_{k n}+\mathrm{i} \epsilon}\right] .
$$

\section{A. Molecule near a plane surface}

For example, let us consider a molecule at a distance $z_{A}$ from the planar surface of a (non-magnetic) substrate. The respective scattering Green tensor is given by [20]

$$
\begin{aligned}
& \mathbf{G}^{(1)}(\boldsymbol{r}, \boldsymbol{r}, \omega)=\frac{\mathrm{i}}{8 \pi} \int_{0}^{\infty} \mathrm{d} q \frac{q}{\beta} e^{2 i \beta z} \\
& \times\left[\left(r_{s}-\frac{\beta^{2} c^{2}}{\omega^{2}} r_{p}\right)\left(\boldsymbol{e}_{x} \boldsymbol{e}_{x}+\boldsymbol{e}_{y} \boldsymbol{e}_{y}\right)+2 \frac{q^{2} c^{2}}{\omega^{2}} r_{p} \boldsymbol{e}_{z} \boldsymbol{e}_{z}\right]
\end{aligned}
$$

where

$$
r_{s}=\frac{\beta-\beta_{1}}{\beta+\beta_{1}}, \quad r_{p}=\frac{\varepsilon(\omega) \beta-\beta_{1}}{\varepsilon(\omega) \beta+\beta_{1}}
$$

with $\operatorname{Im} \beta, \operatorname{Im} \beta_{1} \geq 0$ are the Fresnel reflection coefficients for $s$ - and $p$-polarised waves, $\beta=\sqrt{\omega^{2} / c^{2}-q^{2}}$ and $\beta_{1}=\sqrt{\varepsilon(\omega) \omega^{2} / c^{2}-q^{2}}$ are the $z$-components of the wave vectors in free space and inside the substrate, and $\varepsilon(\omega)$ is the (relative) permittivity of the substrate. Substitution of $\mathbf{G}^{(1)}(\boldsymbol{r}, \boldsymbol{r}, \omega)$ into Eq. (6) above leads to an explicit form for the CP force.

The results simplify in the non-retarded and retarded limits of small and large atom-surface separations. In the non-retarded limit $\max _{i}\left\{\left|\sqrt{\varepsilon\left(\omega_{i}\right)}\right| \omega_{i}\right\} z_{A} / c \ll 1\left(\omega_{i}\right.$ : relevant molecular and medium frequencies), the approximation $\beta \simeq \beta_{1} \simeq \mathrm{i} q$ leads to

$$
\begin{gathered}
\boldsymbol{F}_{n}\left(\boldsymbol{r}_{A}\right)=-\frac{3 k_{\mathrm{B}} T}{8 \pi \varepsilon_{0} z_{A}^{4}} \sum_{N=0}^{\infty}\left(1-\frac{1}{2} \delta_{N 0}\right) \alpha_{n}\left(\mathrm{i} \xi_{N}\right) \frac{\varepsilon\left(\mathrm{i} \xi_{N}\right)-1}{\varepsilon\left(\mathrm{i} \xi_{N}\right)+1} \boldsymbol{e}_{z} \\
-\frac{1}{8 \pi \varepsilon_{0} z_{A}^{4}} \sum_{k}\left|\boldsymbol{d}_{n k}\right|^{2}\left\{\Theta\left(\omega_{n k}\right)\left[n\left(\omega_{n k}\right)+1\right] \frac{\left|\varepsilon\left(\omega_{n k}\right)\right|^{2}-1}{\left|\varepsilon\left(\omega_{n k}\right)+1\right|^{2}}\right. \\
\left.-\Theta\left(\omega_{k n}\right) n\left(\omega_{k n}\right) \frac{\left|\varepsilon\left(\omega_{k n}\right)\right|^{2}-1}{\left|\varepsilon\left(\omega_{k n}\right)+1\right|^{2}}\right\} \boldsymbol{e}_{z} .
\end{gathered}
$$


Note that while applying well to dielectrics, the nonretarded limit often provides a very poor approximation for metals, because the large factor $\left|\sqrt{\varepsilon\left(\omega_{i}\right)}\right|$ may restrict its range of applicability to extremely small distances.

In the retarded limit $\omega_{\min } z_{A} / c \gg 1\left(\omega_{\min }:\right.$ minimum of the relevant molecular and medium frequencies), the resonant part of the force is well approximated by letting $q \simeq 0$, while the approximations $\alpha_{n}\left(\mathrm{i} \xi_{N}\right) \simeq \alpha_{n}(0)$ and $\varepsilon\left(\mathrm{i} \xi_{N}\right) \simeq \varepsilon(0)$ hold for those $\xi_{N}$ giving the main contribution to the non-resonant part. The $q$-integral for the $N=1$ term can then be carried out immediately, while those for the remaining part of the sum can be rewritten in a more convenient form by introducing the integration variable $v=\beta c / \xi_{N}$. Performing the sum according to

$$
\sum_{N=1}^{\infty} N^{4} y^{N}=\frac{y^{4}+11 y^{3}+11 y^{2}+1}{(1-y)^{5}},
$$

one finds

$$
\begin{gathered}
\boldsymbol{F}_{n}\left(\boldsymbol{r}_{A}\right)=-\frac{3 k_{\mathrm{B}} T \alpha_{n}(0)}{16 \pi \varepsilon_{0} z_{A}^{4}} \frac{\varepsilon(0)-1}{\varepsilon(0)+1} \boldsymbol{e}_{z} \\
-\frac{k_{\mathrm{B}} T \alpha_{n}(0)}{2 \pi \varepsilon_{0} z_{A}^{4}} \int_{1}^{\infty} \mathrm{d} v v\left[-\frac{v-\sqrt{\varepsilon(0)-1+v^{2}}}{v+\sqrt{\varepsilon(0)-1+v^{2}}}\right. \\
\left.+\left(2 v^{2}-1\right) \frac{\varepsilon(0) v-\sqrt{\varepsilon(0)-1+v^{2}}}{\varepsilon(0) v+\sqrt{\varepsilon(0)-1+v^{2}}}\right] \\
\quad \times \frac{x^{4}\left(e^{-8 v x}+11 e^{-6 v x}+11 e^{-4 v x}+e^{-2 v x}\right)}{\left(1-e^{-2 v x}\right)^{5}} \boldsymbol{e}_{z} \\
+\frac{\mu_{0}}{6 \pi c z_{A}} \sum_{k}\left|\boldsymbol{d}_{n k}\right|^{2}\left\{\Theta\left(\omega_{n k}\right) \omega_{n k}^{3}\left[n\left(\omega_{n k}\right)+1\right]\right. \\
\quad \times \operatorname{Im}\left[e^{2 \mathrm{i} \omega_{n k} z_{A} / c} \frac{\sqrt{\varepsilon\left(\omega_{n k}\right)}-1}{\sqrt{\varepsilon\left(\omega_{n k}\right)}+1}\right] \boldsymbol{e}_{z}-\Theta\left(\omega_{k n}\right) \omega_{k n}^{3} \\
\left.\quad \times n\left(\omega_{k n}\right) \operatorname{Im}\left[e^{2 \mathrm{i} \omega_{k n} z_{A} / c} \frac{\sqrt{\varepsilon\left(\omega_{k n}\right)}-1}{\sqrt{\varepsilon\left(\omega_{k n}\right)}+1}\right] \boldsymbol{e}_{z}\right\}
\end{gathered}
$$

where $x=2 \pi k_{\mathrm{B}} T z_{A} /(\hbar c)$. In particular, for a conductor whose plasma frequency $\omega_{\mathrm{P}}$ is large compared to $\omega_{n k}$ [cf. Eq. (14) below] one has $|\varepsilon| \gg 1$ and the retarded CP force is well approximated by

$$
\begin{aligned}
\boldsymbol{F}_{n}\left(\boldsymbol{r}_{A}\right) & \approx-\frac{3 k_{\mathrm{B}} T \alpha_{n}(0)}{16 \pi \varepsilon_{0} z_{A}^{4}} \boldsymbol{e}_{z}-\frac{k_{\mathrm{B}} T \alpha_{n}(0)}{8 \pi \varepsilon_{0} z_{A}^{4}} \frac{1}{\left(e^{2 x}-1\right)^{4}} \\
\times & {\left[\left(3+6 x+6 x^{2}+4 x^{3}\right) e^{6 x}-\left(9+12 x-16 x^{3}\right) e^{4 x}\right.} \\
+ & \left.\left(9+6 x-6 x^{2}+4 x^{3}\right) e^{2 x}-3\right] \boldsymbol{e}_{z} \\
+ & \frac{\mu_{0}}{6 \pi c z_{A}} \sum_{k}\left|\boldsymbol{d}_{n k}\right|^{2}\left\{\Theta\left(\omega_{n k}\right) \omega_{n k}^{3}\left[n\left(\omega_{n k}\right)+1\right]\right. \\
& \times \sin \left(2 \omega_{n k} z_{A} / c\right) \boldsymbol{e}_{z}-\Theta\left(\omega_{k n}\right) \omega_{k n}^{3} n\left(\omega_{k n}\right) \\
& \left.\times \sin \left(2 \omega_{k n} z_{A} / c\right) \boldsymbol{e}_{z}\right\} .
\end{aligned}
$$

Note that the retarded limit as given above holds for all distances which are sufficiently large with respect to the atomic and medium wavelengths, irrespective of the temperature. When in addition the distance is very large with respect to the thermal wavelength (such that $x \gg$ 1 ), the contribution from the second terms in the above Eqs. (12) and (13) vanishes and the non-resonant force approaches its well-known (geometric) high-temperature limit, cf. Eq. (1). In the opposite case of a distance which is much smaller than the thermal wavelength $(x \ll 1)$, the first terms vanish and the non-resonant force reduces to its (retarded) zero-temperature form, cf. Ref. 21]. Our results, in particular those for the non-retarded limit, agree with the ones previously obtained in Ref. [15]. Note that resonant force components and their oscillatory behaviour in the retarded regime were first discussed for excited atoms at zero temperature (cf., e.g., [22, 23]).

The limits reveal that the $\mathrm{CP}$ force follows a $1 / z_{A}^{4}$ power law for non-retarded distances. In the retarded regime, the non-resonant force components again follow an inverse power law whereas the resonant force components give rise to spatially oscillating forces whose amplitude is proportional to $1 / z_{A}$. If present, the resonant force components are dominating over the non-resonant ones, in general. The magnitude of the contributions from various molecular transitions to the force (3) is determined by their dipole matrix elements and frequencies, where Eqs. (10) and (12) together with Eq. (7) imply that the strength of the non-resonant force is roughly proportional to $1 / \omega_{k n}$, while that of the resonant force is governed by $n\left(\omega_{n k}\right)+1$ or $n\left(\omega_{k n}\right)$ in the non-retarded limit and by $\omega_{n k}^{3}\left[n\left(\omega_{n k}\right)+1\right]$ or $\omega_{k n}^{3} n\left(\omega_{k n}\right)$ in the retarded regime. Equations (10) and (12) furthermore show that the force becomes larger for larger permittivity of the surface material and saturates in the high-conductivity limit.

The general results and discussion given above can be easily applied to various polar molecules interacting with different surface materials. The qualitative behaviour of the forces will be similar for all molecules and materials, i.e., a power-law dependence for non-retarded distances will give way to an oscillating force in the retarded regime. The exact magnitude of the force as well as the length scale of the oscillations will depend on the dipole moments and frequencies associated with the specific molecular transitions involved and the electric response of the surface in the way indicated above. Tabulated data for a variety of molecules and metal surfaces can be found in Ref. 24]. In the following, we will consider two representative examples.

\section{B. Examples: LiH and YbF near an Au surface}

We first consider a $\mathrm{LiH}$ molecule in its electronic, vibrational, and rotational ground state $\left(p_{n}=\delta_{n 0}\right)$ near an $\mathrm{Au}$ surface at room temperature $T=300 \mathrm{~K}$. With the help of the Green tensor (8), we are able to compute the force components according to Eq. (6) which are displayed in Fig. 1] For this molecule, the contribution from rotational transitions with $\omega_{k n}=2.79 \times 10^{12} \mathrm{rad} / \mathrm{s}$ and $d=1.96 \times 10^{-29} \mathrm{Cm}[24]\left(\sum_{k} \boldsymbol{d}_{0 k} \boldsymbol{d}_{k 0}=d^{2} \mathbf{I}\right.$ where $\mathbf{I}$ is 


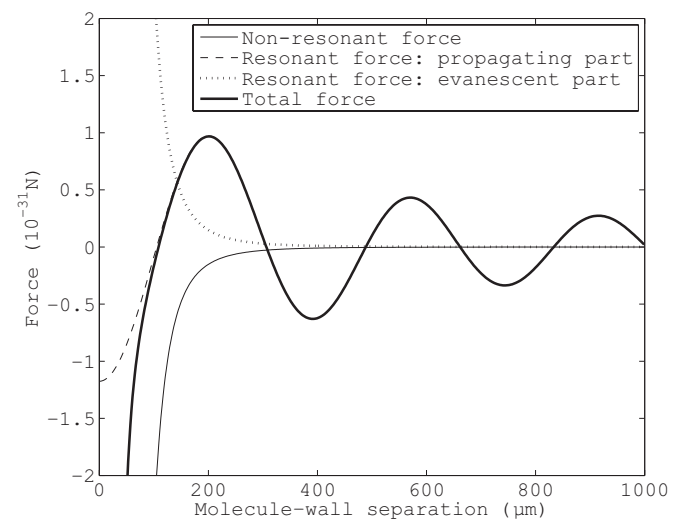

FIG. 1: Thermal CP force on a ground-state LiH molecule near an Au surface. For details, see text.

the unit tensor) is strongly dominant over those of vibrational and electronic transitions with their considerably higher transition frequencies. Molecules with a similar behaviour include NH, OH, OD, NaCs and KCs. For the relative permittivity of the Au surface we have used a Drude model

$$
\varepsilon(\omega)=1-\frac{\omega_{\mathrm{P}}^{2}}{\omega(\omega+\mathrm{i} \gamma)}
$$

with $\omega_{\mathrm{P}}=1.37 \times 10^{16} \mathrm{rad} / \mathrm{s}$ and $\gamma=5.32 \times 10^{13} \mathrm{rad} / \mathrm{s}$ [25]. In view of the current debate regarding the thermal Casimir force (cf. 26, 27] and references therein), we have also calculated the force using the alternative plasma model and found that the difference between the two models is of no importance in our case.

Figure 1 shows the contributions from the non-resonant force component (thin solid line) which is seen to be strictly attractive, and the resonant force components (dashed and dotted lines). With regard to the latter, we have separately shown the propagating part $(q \in[0, \omega / c)$ in Eq. (8), dashed line) and the evanescent part $(q \in[\omega / c, \infty)$, dotted line). The rather astonishing result is that the evanescent part of the resonant force almost exactly cancels the non-resonant force component. Hence, in this highly non-equilibrium situation the largest contribution to the $\mathrm{CP}$ force arises from the propagating part of the resonant force. The total force (thick solid line in Fig. 11) thus closely follows the latter. Only at very small molecule-wall separation $z$ the force is given by its near-field part which, for a two-level isotropic molecule with $\hbar \omega_{A} \ll k_{B} T$, reads

$$
\begin{aligned}
\boldsymbol{F}\left(\boldsymbol{r}_{A}\right) & =\frac{\left|\boldsymbol{d}_{A}\right|^{2}}{8 \pi \varepsilon_{0} z_{A}^{4}}\left[n\left(\omega_{A}\right) \frac{\left|\varepsilon\left(\omega_{A}\right)\right|^{2}-1}{\left|\varepsilon\left(\omega_{A}\right)+1\right|^{2}}-\frac{k_{B} T}{\hbar \omega_{A}} \frac{\varepsilon(0)-1}{\varepsilon(0)+1}\right] \boldsymbol{e}_{z} \\
& \approx \frac{\left|\boldsymbol{d}_{A}\right|^{2}}{8 \pi \varepsilon_{0} z_{A}^{4}}\left[n\left(\omega_{A}\right)-\frac{k_{B} T}{\hbar \omega_{A}}\right] \boldsymbol{e}_{z} .
\end{aligned}
$$

The approximation in the second line of Eq. (15) holds for good conductors. The force saturates in the hightemperature limit where the factor in square brackets ap-

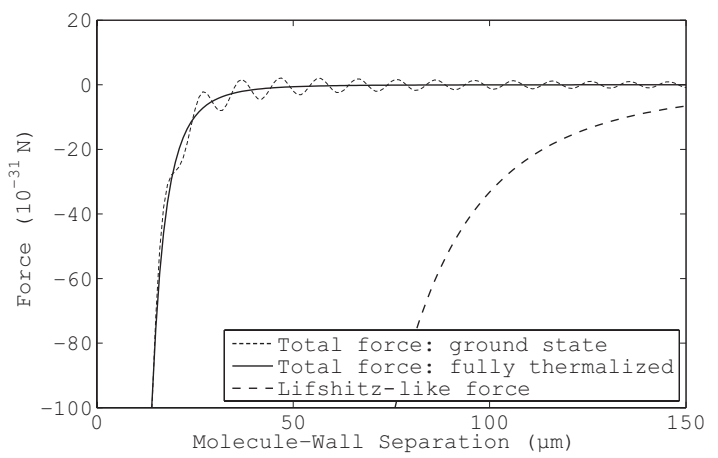

FIG. 2: Thermal CP force on a ground-state vs fully thermalised $\mathrm{YbF}$ molecule near an $\mathrm{Au}$ surface. For details, see text.

proaches $-1 / 2$. In contrast, the non-resonant (Lifshitzlike) force alone would formally diverge. The predicted high-temperature saturation agrees with the previously found vanishing of the leading, linear contribution in $k_{B} T /\left(\hbar \omega_{A}\right)$ in the good-conductor limit [15].

Let us next consider a molecule that is at thermal equilibrium with its environment, so that the probabilities $p_{n}$ are given by a Boltzmann distribution,

$$
p_{n}=\frac{e^{-\hbar \omega_{n} /\left(k_{B} T\right)}}{\sum_{j} e^{-\hbar \omega_{j} /\left(k_{B} T\right)}} .
$$

Here, all resonant force components cancel and the force is given by single a non-resonant force contribution given by the first term in Eq. (6) where the molecular polarisability has to be replaced by its thermal counterpart [18]

$$
\alpha_{T}(\omega)=\sum_{n} p_{n} \alpha_{n}(\omega)
$$

In Fig. 2, we compare this equilibrium force on a thermalised molecule (solid line) with the non-equilibrium ground-state force (dotted line) for the case of YbF. In contrast to $\mathrm{LiH}$, both rotational $\left(\omega_{k n}=9.05 \times 10^{10} \mathrm{rad} / \mathrm{s}\right.$, $\left.d=1.31 \times 10^{-29} \mathrm{Cm}\right)$ and vibrational transitions $\left(\omega_{k n}=\right.$ $9.54 \times 10^{13} \mathrm{rad} / \mathrm{s}, d=8.60 \times 10^{-31} \mathrm{Cm}$ ) [24] give relevant contributions to the force, because at room temperature the frequency of the latter is very close to the peak of the spectrum $\omega_{k n}^{3} n\left(\omega_{k n}\right)$ determining the resonant force contributions in the retarded limit. The results for $\mathrm{YbF}$ are thus representative of those to be expected for $\mathrm{CaF}, \mathrm{BaF}$, $\mathrm{LiRb}, \mathrm{NaRb}, \mathrm{LiCs}$, which also have considerable contributions from vibrational transitions at room temperature. Figure 2 shows that in contrast to the ground-state force, which oscillates as a function of molecule-wall separation (due to the influence of vibrational transitions), the force on a fully thermalised atom is monotonous and attractive (dominated by rotational transitions). We emphasise that the force at thermal equilibrium between the atom and its environment (solid line) is vastly overestimated by a Lifshitz-type macroscopic calculation (dashed 
line) that uses the ground-state polarisability $\alpha_{0}(\omega)$ as input parameter. The reduction factor in the near-field limit is approximately given by [18] as

$$
\frac{|\boldsymbol{F}|}{\left|\boldsymbol{F}_{\text {Lifshitz }}\right|} \simeq \frac{1}{2 n\left(\omega_{10}\right)+1}
$$

for all $\boldsymbol{r}_{A}$. Its dependence on the relevant transition frequency clearly makes it species-dependent. The potentially very large reduction factors $(\approx 1 / 870$ for $\mathrm{YbF}$ at room temperature) imply that these molecules can be brought much closer to metallic surfaces than previously thought.

\section{DYNAMICAL CASIMIR-POLDER FORCE}

In order to understand the transition between the nonequilibrium ground-state force and the fully thermalised one, we need to investigate the full internal molecular dynamics in the presence of the Au surface. The timedependent probabilities $p_{n}=p_{n}(t)$ are governed by the rate equations

$$
\dot{p}_{n}(t)=-\sum_{k} \Gamma_{n k} p_{n}(t)+\sum_{k} \Gamma_{k n} p_{k}(t)
$$

where the transition rates are given by 24 ]

$$
\begin{aligned}
\Gamma_{n k}=\frac{2 \mu_{0}}{\hbar} \omega_{n k}^{2}\{ & \left.\Theta\left(\omega_{n k}\right)\left[n\left(\omega_{n k}\right)+1\right]+\Theta\left(\omega_{k n}\right) n\left(\omega_{k n}\right)\right\} \\
& \times \boldsymbol{d}_{n k} \cdot \operatorname{Im} \mathbf{G}\left(\boldsymbol{r}_{A}, \boldsymbol{r}_{A},\left|\omega_{n k}\right|\right) \cdot \boldsymbol{d}_{k n} \cdot \quad(20)
\end{aligned}
$$

The transition rates for $\mathrm{LiH}$ near an $\mathrm{Au}$ surface can easily be calculated using the Green tensor (8). The resulting time-dependent probabilities $p_{n}(t)$ are displayed for the ground state and the first manifold of rotationally excited states in the lower panels in Fig. 3. with the respective transition matrix elements being given by $\boldsymbol{d}_{|0,0\rangle \rightarrow|1, M\rangle}=d \boldsymbol{u}_{M}, \boldsymbol{u}_{0}=\boldsymbol{e}_{z} / \sqrt{3}, \boldsymbol{u}_{ \pm 1}=\left(\mp \boldsymbol{e}_{x}+\mathrm{i} \boldsymbol{e}_{y}\right) / \sqrt{6}$ [24]. For large molecule-wall separation the transition rates to the different substates of the first manifold are very similar and so are the resulting probabilities (lower right panel). When moving closer to the surface, the transition rates become affected by the evanescent and propagating parts of the reflected field. The contributions of the latter are strongly oscillating so that the rates $\Gamma_{|0,0\rangle \rightarrow|1, \pm 1\rangle}$ exhibit a pronounced minimum at $z=11 \mu \mathrm{m}$. This is not the case for the rate $\Gamma_{|0,0\rangle \rightarrow|1,0\rangle}$ due to the $1 / z$-contribution from the evanescent fields (lower left panel in Fig. 3). Hence, at first only the occupation of the level $|1,0\rangle$ reaches equilibrium with the level $|0,0\rangle$, and full thermalisation is realised only at a much later time.

The dynamics of the $\mathrm{CP}$ force is then governed by the internal molecular dynamics according to

$$
\boldsymbol{F}\left(\boldsymbol{r}_{A}, t\right)=\sum_{n} p_{n}(t) \boldsymbol{F}_{n}\left(\boldsymbol{r}_{A}\right)
$$
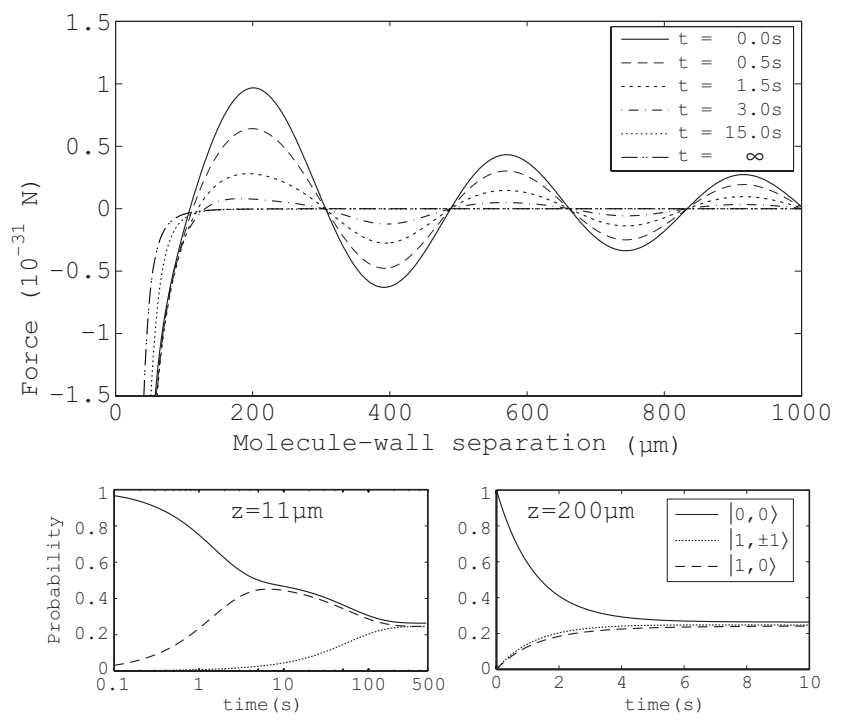

FIG. 3: Transient CP force and internal dynamics of a $\mathrm{LiH}$ molecule initially prepared in its ground state. For details, see text.

This time-dependent force is shown for a $\mathrm{LiH}$ molecule initially prepared in its ground state $p_{n}(t=0)=\delta_{n 0}$ in the top panel of Fig. 3 . We observe a gradual disappearance of the oscillating force components on a timescale of approximately $3 \mathrm{~s}$. The attractive near-field force reaches its equilibrium value only much later due to the above mentioned strongly reduced rate $\Gamma_{|0,0\rangle \rightarrow|1, \pm 1\rangle}$. Note that during the thermalisation the molecule is in an anisotropic state so that we have to use the general expression (3) for the force components rather than its isotropic special case (6).

\section{CONCLUSIONS AND OUTLOOK}

Studying the CP force on polar molecules near a planar surface at finite temperature, we have found that even ground-state molecules are subject to resonant spatially oscillating force components at finite temperature. They are due to the thermal non-equilibrium between the molecule and its environment. A full dynamical treatment has shown that these transient forces disappear in the course of thermalisation of the molecule. The remaining equilibrium force can be vastly different from that calculated using a Lifshitz-type force expression for ground-state molecules.

In our numerical example of ground-state $\mathrm{LiH}$, we have explicitly shown that the non-resonant force component and the evanescent part of the resonant force component cancel almost exactly, leaving a strongly reduced attractive force in the non-retarded limit which saturates at high temperatures. The force in the retarded limit is dominated by resonant contributions from rotational transitions. In contrast, the force on the heavier molecule $\mathrm{YbF}$ is dominated by resonant contributions from vibra- 
tional transitions. Moreover, in thermal equilibrium at room temperature the resulting force is a factor $1 / 870$ smaller than would be expected from a Lifshitz-type calculation for the corresponding ground-state molecule.

Whereas the CP force on a fully thermalised molecule is always attractive, the non-equilibrium force on a ground-state molecule near an Au surface at room temperature has been found to show an oscillating behaviour as a function of the molecule-wall separation $z_{A}$, with stable equilibrium positions away from the surface. Therefore, one might be tempted to use these (transient) minima for trapping purposes. It turns out, however, that for $\mathrm{LiH}$ the first potential well (with its minimum at $z_{A}=300 \mu \mathrm{m}$ ) has a depth of approximately $10^{-12} \mathrm{~K}$ which is immeasurably small. In order to increase the trap depth, one might envisage a situation in which the molecule is embedded in a planar cavity of size $l$ consisting of two such Au surfaces. Then, the Fresnel reflection coefficients in Eq. (8) have to be replaced by $\tilde{r}_{s, p}=r_{s, p} /\left(1-r_{s, p}^{2} e^{2 \mathrm{i} \beta l}\right)$. For very good conductors such as $\mathrm{Au}$, one can set $\left|r_{s, p}\right| \approx 1-\eta$ with $\eta \ll 1$. Hence, for $\beta l=n \pi(n \in \mathbb{N})$, the modified Fresnel coefficients increase as $\left|\tilde{r}_{s, p}\right| \propto 2 / \eta$. Choosing $l$ close to a cavity resonance $n \pi c / \omega_{A}$, the contribution from propagating modes with small $q$ can thus be boosted by several orders of magnitude. Thus, if by use of a cavity with a high $Q$-factor could increase the trap depth by a factor e.g. $10^{6}$, the energy difference would be in the microkelvin regime which could be sufficiently deep to trap cold polar molecules with thermal photons. This question will be addressed in more detail in a future investigation.

\section{Acknowledgments}

This work was supported by the Alexander von Humboldt Foundation and the UK Engineering and Physical Sciences Research Council. S.Y.B. is grateful to M.R. Tarbutt for discussions.
[1] J.J. Hudson, B.E. Sauer, M.R. Tarbutt, and E.A. Hinds, Phys. Rev. Lett. 89, 023003 (2002).

[2] E.D. Commins, Adv. At. Mol. Opt. Phys. 40, 1 (1999).

[3] M.R. Tarbutt, H.L. Bethlem, J.J. Hudson, V.L. Ryabov, V.A. Ryzhov, B.E. Sauer, G. Meijer, and E.A. Hinds, Phys. Rev. Lett. 92, 173002 (2004).

[4] S.Y.T. van de Meerakker, H.L. Bethlem, and G. Meijer, Nature Physics 4, 595 (2008).

[5] S.A. Meek, H.L. Bethlem, H. Conrad, and G. Meijer, Phys. Rev. Lett. 100, 153003 (2008).

[6] S.K. Tokunaga, J.O. Stack, J.J. Hudson, B.E. Sauer, E.A. Hinds, and M.R. Tarbutt, J. Chem. Phys. 126, 124314 (2007).

[7] H.B.G. Casimir and D. Polder, Phys. Rev. 73, 360 (1948).

[8] E.M. Lifshitz, Zh. Eksp. Teor. Fiz. 29, 94 (1955) [Sov. Phys. JETP 2, 73 (1956)].

[9] M. Boström and B.E. Sernelius, Phys. Rev. A 61, 052703 (2000).

[10] J.F. Babb, G.L. Klimchitskaya, and V.M. Mostepanenko, Phys. Rev. A 70042901 (2004).

[11] K.A. Milton, J. Phys. A: Math. Gen. 37, R209 (2004).

[12] A.D. McLachlan, Proc. R. Soc. Lond. Ser. A 274, 80 (1963).

[13] C. Henkel, K. Joulain, J.-P. Mulet, and J.-J. Greffet, J. Opt. A: Pure Appl. Opt. 4, S109 (2002).
[14] T. Nakajima, P. Lambropoulos, and H. Walther, Phys. Rev. A 56, 5100 (1997).

[15] M.-P. Gorza and M. Ducloy, Eur. Phys. J. D 40343 (2006).

[16] M. Antezza, L.P. Pitaevskii, and S. Stringari, Phys. Rev. Lett. 95, 113202 (2005).

[17] J.M. Obrecht, R.J. Wild, M. Antezza, L.P. Pitaevskii, S. Stringari, and E.A. Cornell, Phys. Rev. Lett. 98, 063201 (2007).

[18] S.Y. Buhmann and S. Scheel, Phys. Rev. Lett. 100, 253201 (2008).

[19] Y. Sherkunov, Phys. Rev. A 79, 032101 (2009).

[20] M.S. Tomaš, Phys. Rev. A 51, 2545 (1995).

[21] S.Y. Buhmann, D.-G. Welsch, and T. Kampf, Phys. Rev. A 72, 032112 (2005).

[22] G. Barton, Proc. R. Soc. Lond. Ser. A 320, 251 (1970).

[23] J.M. Wylie and J.E. Sipe, Phys. Rev. A 32, 2030 (1985).

[24] S.Y. Buhmann, M.R. Tarbutt, S. Scheel, and E.A. Hinds, Phys. Rev. A 78, 052901 (2008).

[25] A. Lambrecht and S. Reynaud S, Eur. Phys. J. D 8, 309 (2000).

[26] I. Brevik, S.A. Ellingsen and K.A. Milton, New J. Phys. 8, 236 (2006).

[27] G.L. Klimchitskaya and V.M. Mostepanenko, Contemp. Phys. 47, 131 (2006). 